\title{
Prevalence of thyroid malignancy and hormonal dysfunction following radiation exposure in childhood
}

Yee Sian Tiong *1 ${ }_{M B B S}$, Edwin Tong Yuen Hao *2, Chia Ching $\underline{\text { Lee }}^{3}{ }_{M D}$, Rajeev Parameswaran ${ }^{4}{ }_{F R C S}$, Timothy $\underline{\text { Cheo }}^{3}{ }_{\text {RANZCS, }}$ Wei Li Cindy $\underline{\mathrm{Ho}}{ }^{5,6}{ }_{M R C P C H}$, Samantha Peiling Yang ${ }^{1}{ }_{M R C P}$

\begin{abstract}
Introduction: Childhood radiation exposure is a known risk factor for thyroid malignancy and dysfunction. However, local data are limited and there is no consensus on the modality and frequency of screening in this high-risk group.

Methods: Retrospective analysis study evaluating patients with childhood radiation exposure in 2006-2016 and minimum of 1-year follow-up.

Results: Of the 132 childhood cancer survivors in the study, thyroid malignancy was detected in 2 cases $(1.5 \%)$ and thyroid nodules in $13(9.8 \%)$. The earliest thyroid malignancy was detected 5 years post-radiotherapy via ultrasound. Of the 84 patients who had screening thyroid function test, 26 (31.0\%) were detected with abnormal test results post-radiation, majority being subclinical hypothyroidism.

Conclusion: Regular screening via clinical examination for thyroid nodules should be performed at least annually. Where feasible and if resources permit, consideration should be given to using ultrasound for thyroid nodule(s) and malignancy screening at 5 years post-radiation therapy. Screening for thyroid dysfunction can be considered from 6-12 months post-radiotherapy.
\end{abstract}

Ann Acad Med Singap 2021;50:402-10

Keywords: Childhood post-radiotherapy complications, post-radiotherapy secondary malignancies, thyroid cancer, thyroid dysfunction, thyroid nodules

\section{INTRODUCTION}

The incidence of thyroid cancer has been steadily increasing worldwide. Childhood radiation exposure is a known risk factor for thyroid malignancy. However, data on the incidence of thyroid cancer in this high-risk cohort in Singapore are limited. Thus, we assessed data from patients treated in our institution with a history of childhood radiation exposure over a 10-year period to ascertain the prevalence of thyroid nodules and malignancy, as well as the time interval to the development of thyroid nodules/malignancy and thyroid disease.

\section{Background}

Multiple previous studies have shown that childhood radiation exposure is a risk factor for the development of thyroid malignancy with a summary excess relative risk of 7.7 for every 100 centigray (cGy) of radiation, up to $3,000 \mathrm{cGy}{ }^{1-3,17}$ However, there is a lack of high grade evidence to indicate if early detection of thyroid malignancy improves the quality of life or event-free survival rates in these patients. Hence, there is currently no strong recommendations in the 2015 American Thyroid Association (ATA) regarding thyroid nodule/ cancer screening in this high-risk group. ${ }^{4}$ The current

\footnotetext{
${ }^{1}$ Department of Endocrinology, National University Health System, Singapore

${ }^{2}$ University of Aberdeen, United Kingdom

${ }^{3}$ Department of Radiation Oncology, National University Health System, Singapore

${ }^{4}$ Department of Endocrine Surgery, National University Health System, Singapore

${ }^{5}$ Khoo Teck Puat - National University Children's Medical Institute, National University Health System, Singapore

${ }^{6}$ Department of Paediatrics, Yong Loo Lin School of Medicine, National University of Singapore, Singapore

Correspondence: Dr Wei Li Cindy Ho, Khoo Teck Puat-National University Children's Medical Institute, National University Hospital,

5 Lower Kent Ridge Road, Singapore 119074.

Email: cindy_ho@nuhs.edu.sg

* Joint first authors
} 


\section{CLINICAL IMPACT}

\section{What is New}

- This retrospective study highlights the prevalence of thyroid malignancy and dysfunction in a high-risk group of childhood cancer survivors.

- The study describes the earliest time to development of post-radiation exposure thyroid malignancy or thyroid dysfunction.

\section{Clinical Implications}

- The data in this study can assist in the development of guidelines for the screening and early detection of thyroid malignancy and dysfunction post-radiation exposure in childhood in Singapore.

- This study highlights the importance of regular screening for potential thyroid malignancy or hormonal dysfunction post-radiation exposure in childhood.

- Ultrasound is recommended over palpation to screen patients for thyroid malignancy.

suggestion is for annual clinical examination and the panel "can neither recommend for nor against routine screening ultrasound until more data become available".4 The 2016 American Association of Clinical Endocrinologists (AACE), American College of Endocrinology (ACE) and Associazione Medici Endocrinologi (AME) have recommended more aggressive measures of performing thyroid ultrasound in a patient with risk factors even when the results of palpation are normal. ${ }^{5}$ They have listed a history of head and neck irradiation as a feature suggesting increased risk of malignant potential. ${ }^{5}$ However, they have also not given a latency time post-radiotherapy to start screening this high-risk group, nor a recommendation regarding the interval frequency to perform ultrasound.

Local data on the incidence of thyroid malignancy in childhood cancer survivors with previous radiation exposure are non-existent. Thus, we assessed data from this high-risk population over a 10-year period (20062016) to ascertain the incidence of thyroid nodules and malignancy, the time interval to the development of thyroid nodules or malignancy, and the development of hypothyroidism after radiation exposure.

\section{METHODS}

\section{Subjects}

After obtaining approval from our institutional review board, our team conducted a retrospective analysis of the medical records of 212 patients who received radiotherapy below the age of 20 in 2006-2016. Clinical details such as age, sex, radiation dosages, past history of malignancy, family history of thyroid malignancy or cancer syndromes, treatment, histology findings and image reviewing were collected.

For inclusion in the study, patients had to have undergone radiation exposure for a childhood primary malignancy with a minimum follow-up duration of at least 1 year post-radiation treatment. Of the 212 patients, 132 patients were included and 80 did not meet the study requirement. Of the excluded patients, $68 \%$ were foreigners who were lost to follow-up. The remaining patients had not been followed up for 1 year yet at the time of data collection.

\section{Clinical follow-up}

Currently, there are no clear national guidelines in Singapore for the surveillance of thyroid nodules or thyroid dysfunction in patients who have undergone radiation exposure. Patients were reviewed by their primary treating physicians for underlying malignancy, and surveillance imaging was ordered as per routine clinical guideline specific for the underlying malignancy. In our institution, clinical examination of the thyroid gland was done annually at clinical review where possible. Screening neck ultrasound was performed 2 years post-radiation exposure and follow-up neck ultrasound was conducted every 2 years or earlier if thyroid nodule(s) were detected. Screening neck ultrasound was done regardless of presence or absence of palpable nodules. If any thyroid nodules were detected, the following features were reported including size, composition, echogenicity, shape, margin, presence of echogenic foci and internal vascularity. Thyroid Imaging Reporting and Data System (TIRADs) score was not reported. Screening thyroid function tests (TFTs) were left to the discretion of the primary physician. From 2016, asymptomatic patients had screening TFT 1 year post-radiation and annually thereafter. TFTs were performed earlier in patients who exhibit symptoms or signs suggestive of thyroid dysfunction. Repeat TFT is performed during the next follow-up if an abnormal TFT is detected, depending on how abnormal the initial results were. 


\section{Clinical outcomes}

We aim to assess the adverse clinical outcome of thyroid malignancy arising from childhood radiation exposure. As some of the patients with thyroid nodules may have occult thyroid malignancy, we have included these patients in the data analysis. Records were reviewed until 31 December 2018. We reviewed available imaging modalities including ultrasound, computer tomography, X-ray, positron emission tomography and magnetic resonance imaging for thyroid nodules. Medical records were reviewed for thyroid nodule evaluation and malignancy treatment.

Fine needle aspiration was performed if the thyroid nodule(s) had the following characteristics, including size more than $1 \mathrm{~cm}$, irregular margins, microcalcifications, abnormal cervical lymph nodes, taller than wide, extrathyroidal extension or diffusely infiltrative enlargement of the thyroid lobe. For patients with Bethesda 3-4 cytology results, we followed the 2015 ATA recommendation of hemithyroidectomy in view of the increased probability of malignancy in children. ${ }^{4}$ In patients with non-diagnostic cytology results, we would repeat fine needle aspiration in 3 months.

Treatment response for thyroid cancer was in accordance to the 2015 ATA guidelines.

All TFTs were processed at our institution laboratory using Beckman DXI 680 assay. TFTs measuring the free thyroxine levels (T4) and thyroid-stimulating hormone (TSH) done during the study period were reviewed and categorised in the following subgroups: (1) primary hypothyroidism (low free $\mathrm{T} 4<8.0 \mathrm{pmol} / \mathrm{L}$, elevated $\mathrm{TSH}>4.5 \mathrm{mIU} / \mathrm{L}$ ), (2) central hypothyroidism (low free $\mathrm{T} 4<8.0 \mathrm{pmol} / \mathrm{L}$, inappropriately normal $0.45-4.5 \mathrm{mIU} / \mathrm{L}$ or low $\mathrm{TSH}<0.45 \mathrm{mIU} / \mathrm{L}$ ), and (3) Sub-clinical hypothyroidism (normal free T4 $8.0-16.0 \mathrm{pmol} / \mathrm{L}$, high $\mathrm{TSH}>4.5 \mathrm{mIU} / \mathrm{L})$.

\section{Statistical methods}

Continuous data were presented as median and ranges for each variable. The data were not normally distributed.
Table 1. Characteristics of study patients at baseline

\begin{tabular}{lc}
\hline Patients & $\mathbf{N}=\mathbf{1 3 2}$ \\
\hline Female, n (\%) & $47(36)$ \\
\hline Male, n (\%) & $85(64)$ \\
$\begin{array}{l}\text { Age when first received radiotherapy (years), } \\
\text { median (range) }\end{array}$ & $13.5(1.4-19.9)$ \\
\hline $\begin{array}{l}\text { Follow-up duration (years), median (range) } \\
\text { Dose in cGy, median (range) }\end{array}$ & $4.62(1.02-12.15)$ \\
\hline $\begin{array}{l}\text { Prevalence of thyroid cancer, n (\%) } \\
\text { Prevalence of thyroid nodules, n (\%) }\end{array}$ & $2250(200-6996)$ \\
\hline
\end{tabular}

The chi-square test was used to compare proportions, and Mann-Whitney U Test was used to compare median. Analysis was performed using SPSS Statistics software version 18.0.1 (IBM, Armonk, US). A value of $P \leq 0.05$ was considered statistically significant.

\section{RESULTS}

\section{Baseline clinicopathological characteristics}

Patients were predominantly male (64\%), with a median follow-up duration of 4.62 years (range 1.02-12.15 years) (Table 1).

Most patients had total body irradiation/total nodal irradiation/craniospinal irradiation $(44.0 \%) ; 31.8 \%$ had radiation to the head and neck region; $10.6 \%$ had radiation to the thorax; and $8.3 \%$ had radiation to the abdomen/pelvis (Table 2).

Of the 132 patients, $71(53.8 \%)$ had a primary haematological malignancy; $22.0 \%$ had primary central nervous system malignancy; and $16.6 \%$ had primary musculoskeletal malignancy. None of the patients had a family history of thyroid malignancy nor familial cancer syndromes such as Li-Fraumeni syndrome or Cowden syndrome.

The 80 patients who were excluded from the study had radiotherapy with a median dose of $2,130 \mathrm{cGy}$ (range

Table 2. Distribution of radiation site

\begin{tabular}{|c|c|c|}
\hline Radiation site & $\mathbf{n}$ & $\%$ \\
\hline Total body irradiation/ total nodal irradiation/ craniospinal irradiation & 58 & $44.0 \%$ \\
\hline Head and neck/ central nervous system & 42 & $31.8 \%$ \\
\hline Thorax & 14 & $10.6 \%$ \\
\hline Abdomen/ pelvis & 11 & $8.3 \%$ \\
\hline Extremity & 7 & $5.3 \%$ \\
\hline
\end{tabular}


200-5,940cGy), comparable to the 2,250cGy dose (range 200-6,996cGy) $(P=0.881)$ received by the 132 included patients. There was no significant difference in the age of the 132 patients in the study (median age 13.5 years, 1.4-19.9 years) compared to the 80 excluded patients (median age 11.2 years, 0.6-19.4 years) $(P=0.057)$. Of the excluded subjects, $33.8 \%$ had total body irradiation/total nodal irradiation/craniospinal irradiation, $31.2 \%$ had head/neck region radiation, $13.8 \%$ had thorax radiation, $8.7 \%$ had radiation to abdomen/pelvis and the remainder $12.5 \%$ had radiation to the extremities. We believed that the conclusions drawn from the participants included in study can be applicable to patients with radiation exposure for the treatment of childhood malignancy.

\section{Clinical outcome during follow-up}

In the 132 childhood cancer survivors in the study, the incidence of thyroid nodules is $9.8 \%$ (13/132 cases) and incidence of thyroid malignancy is $1.5 \%(2 / 132$ cases). If we only screened patients who received radiotherapy to the total body, head and neck or thorax region, the incidence of thyroid nodules and malignancy would be higher at $11.4 \%(13 / 114$ cases) and $1.75 \%$ (2/114 cases), respectively.

Table 3 provides the details of the 2 patients diagnosed with papillary thyroid cancer (PTC). Of note, both patients had acute lymphoid leukaemia and underwent bone marrow transplant with total body irradiation.

Table 4 shows a comparison of patients who developed thyroid nodules or malignancy compared to those who did not.

Fig. 1 indicates the proportion of thyroid dysfunction. Of the 132 patients included in the study, 13 patients had thyroid dysfunction prior to radiotherapy. Post-radiotherapy TFT in 6 of these patients had normalised, while 7 patients continued to have abnormalities - 6 with central hypothyroidism and 1 with subclinical hypothyroidism. Five patients with central hypothyroidism had primary brain malignancy.

Of the remaining 119 patients with normal thyroid hormone status prior to radiation therapy, 84 had screening TFT. Twenty-six of these screened (31.0\%) were detected with abnormal test results post-radiation, mainly subclinical hypothyroidism (24/26), whereas

Table 3. Details of patients with thyroid malignancy

\begin{tabular}{|c|c|c|}
\hline & Patient 1 & Patient 2 \\
\hline Sex & Male & Male \\
\hline Primary malignancy & Acute lymphoid leukaemia & Acute lymphoid leukaemia \\
\hline Site of radiation & Total body irradiation & Total body irradiation \\
\hline Total dose of radiation (cGy) & 1200 & 1200 \\
\hline Age when received radiotherapy (years old) & 11 & 5 \\
\hline $\begin{array}{l}\text { Latency period from radiotherapy to diagnosis } \\
\text { of thyroid cancer (years) }\end{array}$ & 9 & 5 \\
\hline Type of thyroid cancer & Papillary thyroid cancer & Papillary thyroid cancer \\
\hline Indication for ultrasound thyroid & Tuberculosis lymphadenitis & Surveillance \\
\hline FNA cytology & $\begin{array}{l}\text { Atypia Follicular Lesion of Undetermined } \\
\text { Significance (Bethesda Class III) }\end{array}$ & Suspicious for PTC (Bethesda V) \\
\hline Histology & $\begin{array}{l}\text { Multifocal PTC with largest } 1.5 \mathrm{~cm} \text { with } \\
\text { extra-thyroidal extension and vascular invasion }\end{array}$ & $\begin{array}{l}\text { Multifocal PTC with lymphovascular } \\
\text { invasion and extrathyroidal extension }\end{array}$ \\
\hline TNM staging & Stage $1 \mathrm{~T} 1 \mathrm{bN} 1 \mathrm{bM} 0$ & Stage $1 \mathrm{~T} 1 \mathrm{~N} 0 \mathrm{Mx}$ \\
\hline Treatment & $\begin{array}{l}\text { Right hemithyroidectomy followed by } \\
\text { completion left thyroidectomy } \\
\text { RAI } 30 \mathrm{mCi}\end{array}$ & $\begin{array}{l}\text { Total thyroidectomy } \\
\text { RAI } 30 \mathrm{mCi}\end{array}$ \\
\hline
\end{tabular}

FNA: fine needle aspiration; PTC: papillary thyroid cancer; RAI: radioiodine; TNM: tumour, nodes and metastases 
Table 4. Comparison of patients with thyroid nodules or malignancy versus no thyroid nodules

\begin{tabular}{|c|c|c|c|}
\hline & $\begin{array}{l}\text { Thyroid nodules/malignancy } \\
\qquad \mathbf{n}=\mathbf{1 3}\end{array}$ & $\begin{array}{l}\text { No thyroid nodules } \\
n=119\end{array}$ & $P$ value \\
\hline $\begin{array}{l}\text { Time post-radiotherapy to detection of noules, } \\
\text { months (range) }\end{array}$ & $14-122$ & - & - \\
\hline Radiation dose cGy, median (range) & $3000(200-6000)$ & $3000(200-7996)$ & 0.606 \\
\hline Euthyroid patients, $\mathrm{n}$ & 6 & 52 & 0.09 \\
\hline Hypothyroid patients, $n$ & 7 & 19 & 0.09 \\
\hline \multicolumn{4}{|l|}{ Site of radiation } \\
\hline TBI, n (\%) & $7(12)$ & $51(88)$ & \\
\hline Head/neck radiation, $\mathrm{n}(\%)$ & $4(9.5)$ & $38(90.5)$ & \\
\hline Thorax, n (\%) & $2(14)$ & $12(86)$ & \\
\hline Abdomen/ pelvis, n (\%) & 0 & $18(100)$ & \\
\hline
\end{tabular}

TBI: total body irradiation

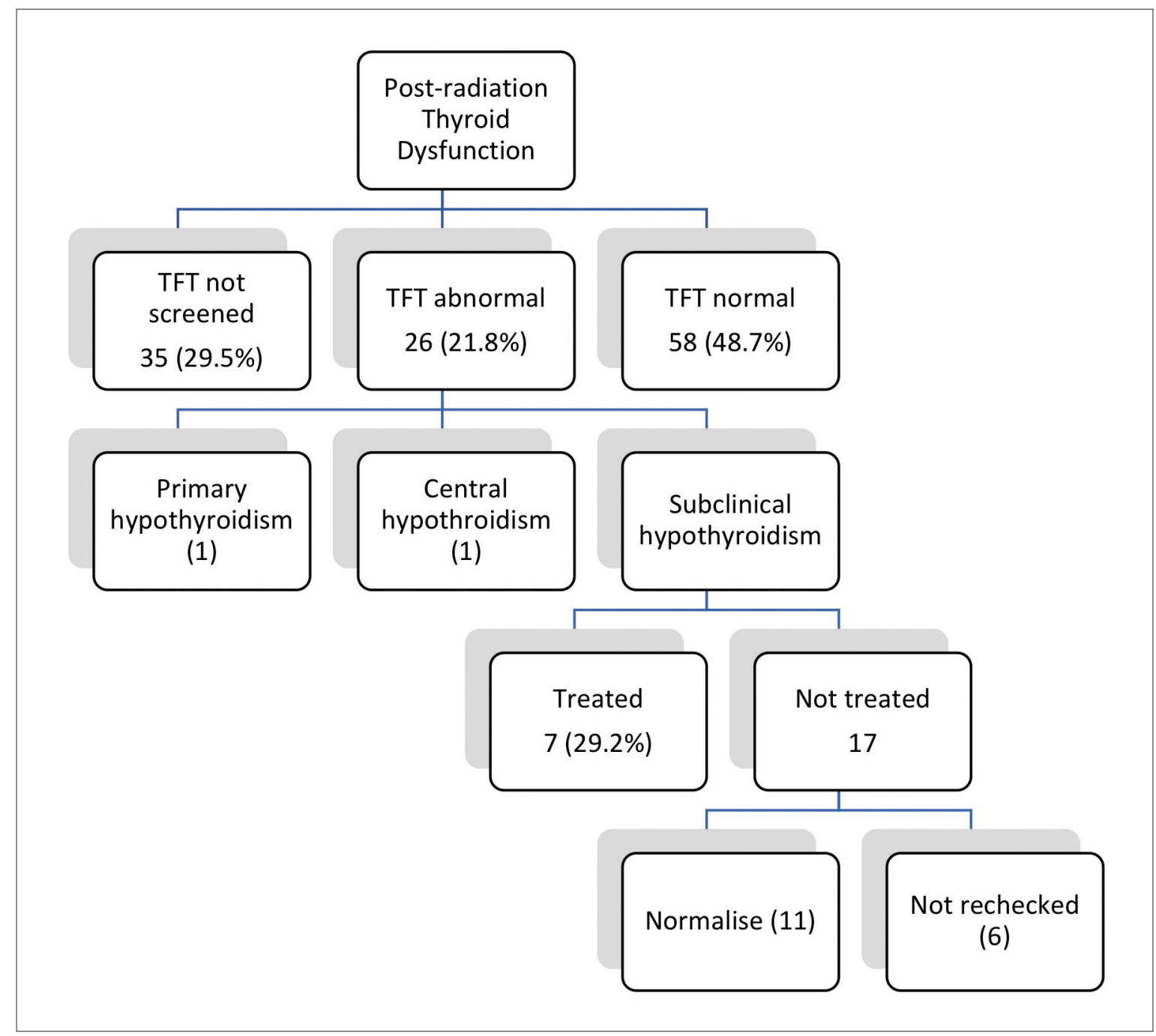

Fig. 1. Post-radiation thyroid dysfunction: Primary hypothyroidism (free $\mathrm{T} 4 \mathrm{of}<8.0 \mathrm{pmol} / \mathrm{L}$ and TSH $>4.5 \mathrm{mlU} / \mathrm{L}$ ); central hypothyroidism (free $\mathrm{T} 4<8.0 \mathrm{pmol} / \mathrm{L}$ and TSH $<4.5 \mathrm{mlU} / \mathrm{L}$ ); subclinical hypothyroidism (free T4 $8-16 \mathrm{pmol} / \mathrm{L}$ and $\mathrm{TSH}>4.5 \mathrm{mlU} / \mathrm{L}$ ).

TFT: thyroid function test 
1 had primary hypothyroidism, and another had central hypothyroidism. Both patients with primary hypothyroidism and central hypothyroidism received thyroxine replacement. Of the 24 patients with subclinical hypothyroidism post-radiotherapy, 7 $(29.2 \%)$ were treated with thyroxine replacement and 11 patients were not treated as their TSH was only marginally elevated. In these 11 untreated patients, their TSH returned to normal spontaneously (Table 5). The remaining 6 had no further TFT done at the completion of the study. Five of the 7 patients treated with levothyroxine had TSH levels $>10 \mathrm{mIU} / \mathrm{L}$. The remaining 2 were started on treatment due to persistently elevated TSH 2 years post-radiotherapy. Interestingly, the patient who had positive antithyroglobulin and anti-thyroid peroxidase antibody results had normal TFT on follow-up 6 months later and thereafter. This could be due to transient thyroiditis in Hashimoto's disease. The correlation between dose of radiation to hypothyroidism achieved statistical clinical significance with a $P$ value of 0.014 . The median dose of patients who remained euthyroid was $1,800 \mathrm{cGy}$ (range 200-7020cGy) compared with 5,580cGy (range 1,200-5,580cGy) in patients who developed hypothyroidism. If patients with radiation to the extremities, abdomen or pelvis was excluded from analysis, the median dose of radiation to the group with normal TFT would be $2,600 \mathrm{cGy}$ with the same dose range of 200-7,020cGy. The $P$ value remains statistically significant at 0.017 . There was also a strong suggestion that the site of radiotherapy (head and neck radiation) is associated with primary hypothyroidism. The patients with thyroid nodules were more likely to have abnormal TFT $(26.9 \%)$ compared to those without thyroid nodules $(10.3 \%)$ (Table 4$)$.

\section{DISCUSSION}

The prevalence of childhood cancer survivors is increasing with a significant proportion having previously undergone radiation therapy. ${ }^{6}$ This group is at a higher risk of developing thyroid disorders and malignancy. Secondary malignancies can occur outside the field of radiation treatment due to scatter radiation, ${ }^{7}$ which includes sites far removed from the initial radiation receiving minute doses of radiotherapy. ${ }^{7,8}$ Therefore, it is important to include patients with no direct thyroid radiation exposure.

In this study, thyroid malignancy was detected in 13\% of survivors with thyroid nodule(s). This is in keeping with the reported incidence of thyroid malignancy of $7.1-40 \%{ }^{1,3,9,10}$ In contrast, the risk of differentiated thyroid malignancy in the general population adults with thyroid nodules is only $5 \% .{ }^{11}$ The incidence of

Table 5. Comparison of patients with persistent versus transient subclinical hypothyroidism

\begin{tabular}{|c|c|c|c|}
\hline & $\begin{array}{l}\text { Persistent } \\
\mathbf{n}=7\end{array}$ & $\begin{array}{l}\text { Normalised } \\
\mathbf{n}=\mathbf{1 1}\end{array}$ & $\begin{array}{l}\text { Not checked } \\
n=6\end{array}$ \\
\hline $\begin{array}{l}\text { Time to development of abnormal TFT } \\
\text { (months), median (range) }\end{array}$ & $14.5(3.4-59.6)$ & $3.7-63.1$ & $4.3-60.7$ \\
\hline TSH range (mIU/L) (first TFT) & $4.67-23.36$ & $4.16-10.19$ & $4.63-6.72$ \\
\hline \multicolumn{4}{|l|}{ Anti-TPO Abs/ Anti-Tg Abs } \\
\hline Negative & 6 & 3 & - \\
\hline Positive & - & 1 & - \\
\hline Not checked & 1 & 7 & - \\
\hline Radiation dose, median (range) & $5580(1200-5580)$ & $800(200-5580)$ & $3840(200-6000)$ \\
\hline$P$ value & 0.023 & & \\
\hline \multicolumn{4}{|l|}{ Site of radiation } \\
\hline TBI & 4 & 9 & 4 \\
\hline Head/neck & 2 & 1 & 2 \\
\hline Thorax & 1 & - & - \\
\hline Abdomen/pelvis/ extremity & - & 1 & - \\
\hline
\end{tabular}

anti-TPO Abs: anti-thyroid peroxidase antibodies; anti-Tg Abs: anti-thyroglobulin antibodies; TBI: traumatic brain injury; TFT: thyroid function test; TSH: thyroid-stimulating hormone 
differentiated thyroid malignancy in the paediatric population is even lower at 0.54 case per 100,000 children. ${ }^{12}$ One of the major differences between thyroid cancer (specifically PTC) diagnosed in childhood and adulthood is the higher proportion of extrathyroidal extension, lymph node and pulmonary metastases, and higher recurrence rate. ${ }^{13-18}$ This is evident in both our patients with PTC. Fortunately, the mortality rate is still low at $2 \%$ or less and there is better progression-free survival compared to adults. . $^{13,14,16,18}$

As the risk of malignancy is up to 20 fold higher compared to the general population, ${ }^{19}$ regular surveillance is important.

In our study, both patients who were diagnosed with PTC received a total of $1,200 \mathrm{cGy}$ of radiation. This result is consistent with a pooled analysis done on the strength of the relationship between external radiation exposure and thyroid carcinoma. ${ }^{20,21}$ The dose-relationship relative risk of thyroid radiation exposure and thyroid carcinoma development is supralinear from 200-400cGy, plateaus at 1,000-3,000cGy and declines after 3,000cGy. ${ }^{20}$ The relative risk remains significantly elevated up to $5,000 \mathrm{cGy} .{ }^{20}$ This is because thyroid tissues that receive higher doses of radiotherapy are more likely to undergo cell death from lethal double-stranded DNA breaks, rather than develop malignant cell transformation into thyroid carcinoma. ${ }^{22}$ We included patients who had no direct thyroid irradiation as scatter radiation is a known risk factor for secondary malignancies. ${ }^{6,17}$

The latency period from radiation exposure to the development of thyroid malignancy is approximately 5-10 years with peak incidence at 25-29 years postexposure. ${ }^{2,21}$ In our cohort, we excluded data from patients who have had radiation therapy within 1 year of analysis of the data due to the low probability of radiation being the direct cause of the thyroid malignancy. In the patients who developed thyroid malignancy, patient 1 and 2 had a time latency of 9 years and 5 years, respectively (Table 3 ). This is concordant with the expected time latency. As we only had data for the past 10 years, this cohort of patients will need to be followed up as we have yet to reach the peak incidence of thyroid nodules and thyroid cancer.

Previous data have indicated that ultrasound is more sensitive in the detection of thyroid nodules compared to palpation. ${ }^{22}$ There is no consensus between the major guidelines of ATA, AME and AACE on screening patients using ultrasound.
The International Guideline Harmonization Group (IGHG) recommendation is to screen high-risk patients with previous radiation therapy directly or indirectly around the thyroid region. ${ }^{23}$ However, IGHG has left the screening modality up to the discretion of the healthcare provider in discussion with the patient. ${ }^{23}$ IGHG also suggests that screening for thyroid nodules or malignancy can start 5 years post-radiotherapy. ${ }^{23}$

A retrospective review of adult survivors of childhood cancer with previous neck radiation involving 585 patients found that only $1.2 \%$ of patients with abnormal physical examination were diagnosed with PTC. ${ }^{24}$ The authors concluded that annual physical examination without routine ultrasound can be used as a screening tool. ${ }^{24}$

In our study, one of our patients with thyroid malignancy was only detected on surveillance ultrasound and not clinically. He had T1bN1bM0 papillary thyroid cancer that could have resulted in a higher morbidity with delayed detection.

Based on our cohort data, clinical assessment for thyroid malignancy using palpation should be performed annually. Subsequently, consideration should be given to performing thyroid ultrasound every 1-2 years from 5 years post-radiotherapy to improve the sensitivity of detecting thyroid nodules. Two main reasons for our suggestion are the latency period of 5 years prior to development of thyroid malignancy post-radiation exposure in our and other cohorts, and to reduce delay in the detection of potentially malignant thyroid nodules.

Thyroid hormone dysfunction is a well-documented complication post-radiation therapy. ${ }^{25-28}$ Primary hypothyroidism is the most common with a prevalence of $13.8-20.8 \% .^{25-27}$ The average time to the development of hypothyroidism have been reported to be 5-7 years (range of $0-27$ years)..$^{25,28}$ In our cohort, 9 out of 84 $(10.7 \%)$ patients who had screening TFTs developed hypothyroidism (Fig. 1). The dose of radiation therapy (higher risk with higher dose) (Table 5) is associated with the development of primary hypothyroidism. Risk factors shown to correlate with the development of primary hypothyroidism include higher radiation doses, proximity to the thyroid gland, and female gender. ${ }^{25,28,29}$ The actual dose-event rate is not well-established as some studies reported total radiation doses and others estimated radiation dose to the thyroid gland. ${ }^{25,28,29}$ We could not determine the actual radiation dose that was delivered to the thyroid gland, thus are unable to derive the "pathogenic" radiation dose. Our sample size was too small to determine if gender is an independent 
risk factor. Additionally, the median follow-up in our sample population was 4.59 years, which likely represents an underestimate of the true hypothyroidism prevalence.

The Children's Oncology Group have advocated for annual (or more frequent) TFT screening in patients at risk of central hypothyroidism or primary hypothyroidism secondary to radiotherapy starting at 2 years postradiotherapy. ${ }^{30}$

Based on our cohort, the risk of thyroid dysfunction is high. Screening TFTs should be performed 6-12 months post-radiotherapy or earlier if the patient develops clinical symptoms or signs suggestive of thyroid dysfunction. Thus, our recommendation allows for more targeted screening and abnormal TFT from non-thyroidal illness to resolve.

Previous cohorts have shown that thyroid dysfunction can occur up to 20 years post-radiation exposure. ${ }^{25}$ Therefore, these high-risk patients will require long-term follow-up to monitor for the development of thyroid dysfunction.

Another limitation of our study stems from a large percentage of patients $(68 \%)$ who were excluded due to lost follow-up, resulting in exclusion bias. Additionally, median follow-up time was short at 4.62 years. It would be worthwhile to re-analyse the data of these patients after a longer period of follow-up.

\section{CONCLUSION}

Screening for thyroid nodules, malignancy and thyroid dysfunction is an important aspect in the long-term follow-up of childhood cancer survivors postradiotherapy. Regular screening via clinical examination for thyroid malignancies should be performed at least annually. Where feasible and if resources permit, consideration should be given to using ultrasound for thyroid nodule(s) and malignancy screening at 5 years post-radiation therapy as it is more sensitive compared to palpation at detecting smaller nodules based on data from our cohort. Screening for thyroid dysfunction in asymptomatic patients should start from 6-12 months post-radiotherapy, as data from our cohort show that it can occur at an earlier interval. Longer term follow-up is required to determine the actual incidence of thyroid malignancy and thyroid dysfunction in this high-risk cohort.

\section{REFERENCES}

1. Ron E, Lubin JH, Shore RE, et al. Thyroid cancer after exposure to external radiation: a pooled analysis of seven studies. Radiat Res 1995;141:259-77.
2. Ho WLC, Zacharin MR. Thyroid carcinoma in children, adolescents and adults, both spontaneous and after childhood radiation exposure. Eur J Pediatr 2016;175:677-83.

3. Acharya S, Sarafoglou K, LaQuaglia M, et al. Thyroid neoplasms after therapeutic radiation for malignancies during childhood or adolescence. Cancer 2003;97:2397-403.

4. Francis GL, Waguespack SG, Bauer AJ, et al. Management guidelines for children with thyroid nodules and differentiated thyroid cancer: The American Thyroid Association guidelines task force on pediatric thyroid cancer. Thyroid 2015;25:716-59.

5. Gharib H, Papini E, Garber JR, et al. American Association of Clinical Endocrinologists, American College of Endocrinology, and Associazione Mdici Endocrinologi medical guidelines for clinical practice for the diagnosis and management of thyroid nodules -2016 update: Appendix. Endocr Pract 2016;22(Supplement 1):1-60.

6. Phillips SM, Padgett LS, Leisenring WM, et al. Survivors of childhood cancer in the united states: prevalence and burden of morbidity. Cancer Epidemiol Biomarkers Prev 2015;24:653-63.

7. Chaturvedi AK, Engels EA, Gilbert ES, et al. Second cancers among 104760 survivors of cervical cancer: evaluation of long-term risk. JNCI J Natl Cancer Inst 2007;99:1634-43.

8. Diallo I, Haddy N, Adjadj E, et al. Frequency distribution of second solid cancer locations in relation to the irradiated volume among 115 patients treated for childhood cancer. Int J Radiat Oncol 2009;74:876-83.

9. de Vathaire F, Hardiman C, Shamsaldin A, et al. Thyroid carcinomas after irradiation for a first cancer during childhood. Arch Intern Med 1999;159:2713-9.

10. Brignardello E, Felicetti F, Castiglione A, et al. Ultrasound surveillance for radiation-induced thyroid carcinoma in adult survivors of childhood cancer. Eur J Cancer 2016;55:74-80.

11. Gharib H, Papini E. Thyroid nodules: clinical importance, assessment, and treatment. Endocrinol Metab Clin North Am 2007;36:707-35

12. Verburg FA, van Santen H, Luster M. Pediatric papillary thyroid cancer: current management challenges. OncoTargets Ther 2016;10:165-75.

13. Chow S-M, Law SCK, Mendenhall WM, et al. Differentiated thyroid carcinoma in childhood and adolescence?clinical course and role of radioiodine. Pediatr Blood Cancer 2004;42:176-83.

14. Newman KD, Black T, Heller G, et al. Differentiated thyroid cancer: determinants of disease progression in patients $<21$ years of age at diagnosis: a report from the surgical discipline committee of the children's cancer group. Ann Surg 1998;227:533-41.

15. Demidchik YE, Demidchik EP, Reiners C, et al. Comprehensive clinical assessment of 740 cases of surgically treated thyroid cancer in children of Belarus. Ann Surg 2006;243:525-32.

16. Jarząb B, Junak DH, Włoch J, et al. Multivariate analysis of prognostic factors for differentiated thyroid carcinoma in children. Eur J Nucl Med Mol Imaging 2000;27:833-41.

17. O'Gorman CS, Hamilton J, Rachmiel M, et al. Thyroid cancer in childhood: a retrospective review of childhood course. Thyroid 2010;20:375-80.

18. Hay ID, Gonzalez-Losada T, Reinalda MS, et al. Long-term outcome in 215 children and adolescents with papillary thyroid cancer treated during 1940 through 2008. World J Surg 2010; 34:1192-202.

19. de Vathaire F, Haddy N, Allodji RS, et al. Thyroid radiation dose and other risk factors of thyroid carcinoma following childhood cancer. J Clin Endocrinol Metab 2015;100:4282-90. 
20. Veiga LHS, Holmberg E, Anderson H, et al. Thyroid cancer after childhood exposure to external radiation: an updated pooled analysis of 12 studies. Radiat Res 2016;185:473-84.

21. Schneider AB, Ron E, Lubin J, et al. Dose-response relationships for radiation-induced thyroid cancer and thyroid nodules: evidence for the prolonged effects of radiation on the thyroid. J Clin Endocrinol Metab 1993;77:362-9.

22. Schneider AB, Bekerman $\mathrm{C}$, Leland $\mathrm{J}$, et al. Thyroid nodules in the follow-up of irradiated individuals: comparison of thyroid ultrasound with scanning and palpation. J Clin Endocrinol Metab 1997;82:4020-7.

23. Clement SC, Kremer LCM, Verburg FA, et al. Balancing the benefits and harms of thyroid cancer surveillance in survivors of childhood, adolescent and young adult cancer: recommendations from the international late effects of childhood cancer guideline harmonization group in collaboration with the PanCareSurFup Consortium. Cancer Treat Rev 2018;63:28-39.

24. Tonorezos ES, Barnea D, Moskowitz CS, et al. Screening for thyroid cancer in survivors of childhood and young adult cancer treated with neck radiation. J Cancer Surviv 2017;11:302-8.
25. Sklar C, Whitton J, Mertens A, et al. Abnormalities of the thyroid in survivors of Hodgkin's disease: data from the childhood cancer survivor study. J Clin Endocrinol Metab 2000;85:3227-32.

26. Brignardello E, Felicetti F, Castiglione A, et al. Endocrine health conditions in adult survivors of childhood cancer: the need for specialized adult-focused follow-up clinics. Eur J Endocrinol. 2013;168:465-72.

27. Hudson MM, Ness KK, Gurney JG, et al. Clinical ascertainment of health outcomes among adults treated for childhood cancer. JAMA 2013;309:2371.

28. Çağlar AA, Oğuz A, Güçlü Pınarlı F, et al. Thyroid abnormalities in survivors of childhood cancer. J Clin Res Pediatr Endocrinol. 2014;144-51.

29. Vogelius IR, Bentzen SM, Maraldo MV, et al. Risk factors for radiation-induced hypothyroidism: A literature-based meta-analysis. Cancer 2011;117:5250-60.

30. Children's Oncology Group. Long-term follow-up guidelines for survivors of childhood, adolescent and young adult cancers, Version 5.0 , 2018. Available at: www.survivorshipguidelines.org. Accessed on 10 December 2020. 